\title{
A Versatile Transfection Assay System to Evaluate the Biological Effects of Diverse Industrial Chemicals
}

\author{
Shinji Koizumi, ${ }^{a}$ Shotaro Ohno, ${ }^{b}$ and Fuminori Otsuka*,b \\ ${ }^{a}$ Department of Research Planning and Coordination, National Institute of Occupational Safety and Health; 6-21-1 \\ Nagao, Tama-ku, Kawasaki 214-8585, Japan: and ${ }^{b}$ Laboratory of Molecular Environmental Health, Department of \\ Life and Health Sciences, Faculty of Pharma-Sciences, Teikyo University; 2-11-1 Kaga, Itabashi-ku, Tokyo 173- \\ 8605, Japan. Received February 19, 2012; accepted July 2, 2012
}

Gene expression processes are now recognized as important targets of the toxic effects exerted by industrial chemicals. The transient transfection assay is a powerful tool to evaluate such effects. Thus, we developed a versatile assay system by constructing a basic reporter plasmid in which the regulatory DNA sequence to be studied can easily be substituted. To verify the performance of this system, reporter plasmids carrying any of the three distinct regulatory sequences, estrogen responsive element (ERE), glucocorticoid responsive element (GRE) and xenobiotic responsive element (XRE) were constructed. After transfection of human cells, these plasmids successfully expressed the relevant reporter genes in response to specific inducers, $\beta$-estradiol, dexamethasone and 3-methylcholanthrene, respectively. Several industrial chemicals were assayed using these reporter plasmids, and the ability of $p$-dimethylaminoazobenzene to elevate GRE- and XRE-mediated transcription was detected. $\alpha$-Naphthylamine and $o$-tolidine were also observed to increase the XRE-mediated response. The transfection assay system established here will be useful to evaluate the effects of a wide variety of industrial chemicals.

Key words estrogen; gene regulation; glucocorticoid; industrial chemical; transient transfection assay; xenobiotic

Several years ago, when endocrine-disrupting chemicals attracted considerable attention as environmental pollutants, it was hypothesized that gene expression processes are potential important targets that mediate the detrimental effects exerted by chemicals. A number of industrial chemicals target gene expression processes, and therefore, methods for the rapid and accurate assessment of their effects on gene regulation are required. The transient transfection assay is a useful tool for this purpose; it can evaluate the effect of a chemical on a specific gene regulation process that is mediated by a distinct regulatory DNA sequence. This assay requires a reporter plasmid, which usually contains a regulatory DNA sequence, promoter, and a downstream-linked reporter gene. Following transfection of cultured cells with the reporter plasmid, cells are exposed to the chemicals to be tested for an amount of time appropriate to each system. The expression levels of the reporter protein in cell lysates are then determined, and this serves as an indicator of the effect on transcription mediated by the regulatory sequence. ${ }^{1)}$ This kind of assay has previously been used to screen endocrine-disruptor activity; however, the effects of many other chemicals remain to be studied. Construction of reporter plasmids specific for individual purposes has been laborious, and this appears to be one of the reasons why this assay has not been widely used for toxicity assessment. In the present study, we describe a versatile transfection assay system using a basic plasmid designed to permit convenient construction of a variety of reporter plasmids. We also present data on the practical use of this system in evaluating the effects of several industrial chemicals on gene expression.

\section{MATERIALS AND METHODS}

Materials HeLa S3 cells, derived from a human cervi-

The authors declare no conflict of interest. cal carcinoma, were obtained from ATCC (Rockville, MD, U.S.A.), and HepG2 cells, derived from human hepatoma, were from RIKEN Cell Bank. MCF-7 cells, derived from human breast carcinoma, were purchased from Dainippon Chemicals Ind., Ltd. (Tokyo, Japan). Sodium pyruvate, nonessential amino acids, Eagle's minimum essential medium (MEM) and Dulbecco's MEM were purchased from Life Technologies Corporation. Fetal calf serum was obtained from biowest (Nuaillé, France) and charcoal/dextran-treated fetal calf serum was from Thermo Fisher Scientific (U.S.A.). Dihydrotestosterone (DHT) was purchased from Wako Pure Chemical Industries, Ltd. (Osaka, Japan). Progesterone (Prog) was obtained from Nacalai Tesque (Kyoto, Japan). Dexamethasone (Dex), $\beta$-estradiol $\left(\mathrm{E}_{2}\right)$ and deoxycorticosterone (DOC) were obtained from Sigma-Aldrich. Auramine, $p$-dimethylaminoazobenzene (DAB), magenta, $\alpha$-naphthylamine, pentachlorophenol (PCP), o-tolidine, toluenediisocyanate (TDI), 1,1,2-trichloroethane (1,1,2-TCE) and 3-methylcolanthlene (3-MC) were purchased from Wako Pure Chemical Industries, Ltd. All other chemicals used were of special grade.

Cell Culture HeLa S3 cells were maintained in Eagle's MEM supplemented with 10\% fetal calf serum. MCF7 cells were maintained in Eagle's MEM supplemented with 10\% fetal calf serum, nonessential amino acids, and $1 \mathrm{~mm}$ sodium pyruvate. HepG2 cells were cultured in Dulbecco's MEM supplemented with $10 \%$ fetal calf serum.

Construction of Reporter and Reference Plasmids The minimal promoter region of the herpes simplex virus thymidine kinase (HSV-TK) gene was amplified by polymerase chain reaction (PCR) using the plasmid $\mathrm{p}(\mathrm{MREa}){ }_{4} \mathrm{TKprCAT}^{2)}$ as a template and the following primer pair: 5'-ATA GGCGTA TCA CGA GCTCCT TTCGTC TTC-3' and 5'-TCTCCA TTT TAGCTTCCTTAGCTCC-3'. The PCR product contained the TK promoter $(-80$ to +57$)$, flanked by BglII and HindIII sites at the 5 '- and 3 '-termini, respectively. This PCR product 
was digested with $B g l \mathrm{II}$ and HindIII, gel-purified, and inserted between the BglII and HindIII sites of the pGL3-Basic plasmid (Promega, U.S.A.) in order to generate pGL3/TK that carries the minimal TK promoter linked to the firefly luciferase reporter gene (Fig. 1). The nucleotide sequence of the inserted promoter region was verified by DNA sequencing. Double-stranded DNA oligonucleotides containing regulatory sequences (chemically synthesized by Sawady Technology, Japan) were designed to have a flanking BamHI site at one end and a BglII site at the other end. The BamHI site was placed at the upstream end of the sequence in the normal orientation (indicated in Figs. 1), and the BglII site at the opposite end. The oligonucleotides containing the estrogen-responsive element (ERE), glucocorticoid-responsive element (GRE), and xenobiotic-responsive element (XRE) were inserted into the BglII site of pGL3/TK; the nucleotide sequences and orientations of the inserts were confirmed by DNA sequencing, and plasmids carrying the correct sequences in the normal orientation were selected and termed pGL3/ERE-TK, pGL3/ (GRE) $)_{2}-\mathrm{TK}$, and pGL3/(XRE) $)_{2} \mathrm{TK}$, respectively (Fig. 1). As a reference plasmid, $\mathrm{pRL} / \mathrm{RSV}$, which carries the Renilla luciferase (RL) gene driven by the Rous sarcoma virus (RSV) promoter, was used. pRL/RSV was constructed by substituting the firefly luciferase gene of $\mathrm{pRSVL}^{2)}$ with the RL gene from $\mathrm{pRL} / \mathrm{TK}$ (Promega, U.S.A.). For transfection experiments, the plasmids were amplified in Escherichia coli DH5 $\alpha$ (Toyobo, Japan), and purified using an EndoFree Plasmid Kit (QIAGEN, Germany).

Transient Transfection Assay For the transfection assay, phenol red-free medium containing $10 \%$ charcoal/dextran-treated fetal calf serum was used. Cells were plated at a density of $10^{5}$ cells in $0.5 \mathrm{~mL}$ medium per well in 24-well plates (Asahi Techno Glass, Japan), and incubated at $37^{\circ} \mathrm{C}$ for $24 \mathrm{~h}$ in an atmosphere of $5 \% \mathrm{CO}_{2}$. A reporter plasmid $(0.5 \mu \mathrm{g})$ and the reference plasmid pRL/RSV ( $2 \mathrm{ng}$ ) were added to the medium as a mixture with FuGENE 6 reagent (Roche Diagnostics, Japan) at a ratio of $1 \mu \mathrm{L}$ reagent: $4 \mu \mathrm{g}$ DNA. After $16 \mathrm{~h}$, cells were washed twice with $\operatorname{PBS}(-)$ (phosphate-buffered saline without $\mathrm{Mg}^{2+}$ and $\mathrm{Ca}^{2+}$ ), and given fresh medium. A few hours later, the chemicals to be tested were added to the culture medium, and the cells were incubated for a further $16 \mathrm{~h}$. The incubation time was modified according to previous reports for related assays. ${ }^{3-6)}$ For determination of expressed firefly luciferase and RL activity, the Dual Luciferase Assay System (Promega, U.S.A.) was used. After washing cells twice with $\operatorname{PBS}(-)$, lysis buffer was added $(100 \mu \mathrm{L} /$ well), and the cells were incubated for $20 \mathrm{~min}$ with shaking at room temperature. The lysate $(2-10 \mu \mathrm{L})$ was mixed with $50 \mu \mathrm{L}$ of the firefly luciferase reaction mixture and the intensity of luminescence was determined by a luminometer (Model TD-20/20, Promega, U.S.A.). The reactions were then supplemented with $50 \mu \mathrm{L}$ of $\mathrm{RL}$ reaction mixture for the second luminescence determination. Reporter (firefly luciferase) activity was normalized to reference (RL) activity. Assays were carried out in duplicate unless otherwise stated and averages are presented. The Student's $t$-test was used for statistical comparisons in Table 1.

Cytotoxicity Testing Cells were plated at a density of $2 \times 10^{4}$ cells in $0.1 \mathrm{~mL}$ medium per well in 96-well plates (Corning, U.S.A.), and incubated for $1 \mathrm{~d}$. Chemicals to be tested were added to the medium and cells were cultured for a

\section{ERE (Xenopus vitellogenin-A2 gene)}

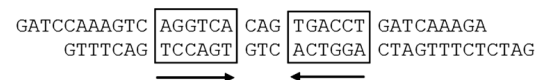

PRE/GRE/ARE/MRE (Rat tyrosine aminotransferase gene)

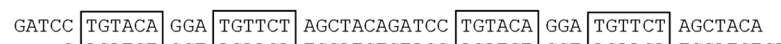

G \begin{tabular}{lllll} 
ACATGT & CCT & ACAAGA & TCGATGTCTAGG & ACATGT \\
\hline
\end{tabular}

\section{XRE (Mouse CYP1A1gene)}

GATCCGCCGGGT TTGCGTG CGATGAGATCCGCCGGGT TTGCGTG CGATGA GCGGCCCA AACGCAC GCTACTCTAGGCGGCCCA AACGCAC GCTACTCTAG

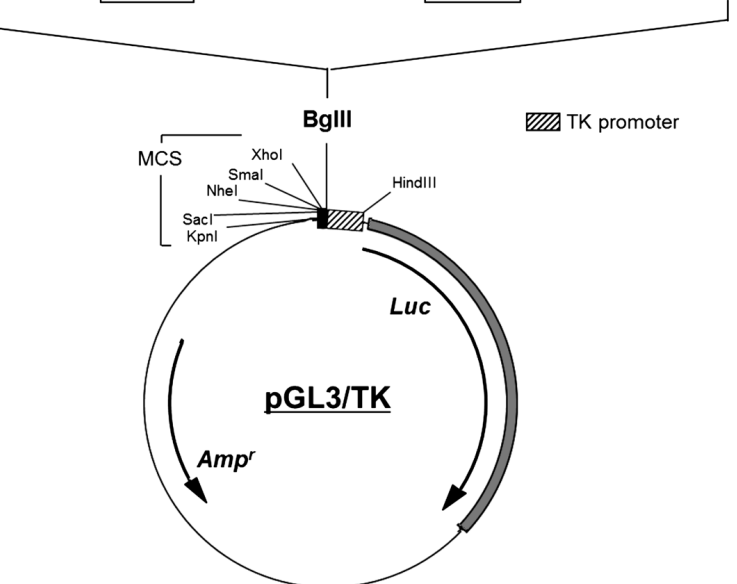

Fig. 1. Structure of Reporter Plasmids

MCS, multiple cloning site; Luc, firefly luciferase gene; Amp ${ }^{\mathrm{r}}$, ampicillin resistance gene. Oligonucleotides containing the regulatory sequences indicated in the upper half of the figure were inserted into the BglII site of the basic reporter plasmid pGL3/TK (4944bp). The resulting plasmids containing ERE, PRE/GRE/ARE MRE or XRE sequences in the indicated orientation were termed pGL3/ERE-TK, pGL3/(GRE) $)_{2}$-TK and pGL3/(XRE) $)_{2}$ TK, respectively.

further $16 \mathrm{~h}$. The medium was replaced after washing 2 times with $\operatorname{PBS}(-)$, and supplemented with $10 \mu \mathrm{L}$ of a tetrazolium salt (WST-1; TaKaRa, Japan). After $30 \mathrm{~min}$, succinate-tetrazolium reductase activity present in viable cell mitochondria was monitored by measuring absorbance at $450 \mathrm{~nm}$ with a microplate reader (Model 550, Bio-Rad Japan).

\section{RESULTS}

Construction of a Versatile Basic Reporter Plasmid To facilitate the otherwise laborious reporter plasmid preparation, we designed and constructed a versatile reporter plasmid, pGL3/TK, into which regulatory DNA sequences can be easily inserted (Fig. 1). pGL3/TK contains the minimal HSV-TK promoter and firefly luciferase reporter gene linked immediately downstream. The TK promoter was chosen as the minimal promoter since it contains sequences essential for transcription, but has very low activity by itself, and this is advantageous for reducing the background level of reporter activity. The multiple cloning site (MCS) was positioned just upstream of the TK promoter, thereby providing insertion sites for the desired regulatory sequences. We used the BglII site as a convenient site for insertion of synthetic oligonucleotides with flanking BamHI and $B g l \mathrm{II}$ sites. Insertion of such oligonucleotides creates $B g l \mathrm{II} / \mathrm{Bam} \mathrm{HI}$ and $B g l \mathrm{II} / B g l \mathrm{II}$ junctions: the former becomes resistant to $B g l \mathrm{II}$ digestion, and the orientation of the insert is easily determined by restriction enzyme analysis. When the $B g l \mathrm{II} / B g l \mathrm{II}$ junction is placed proximal 
to the transcription initiation site (TIS), it can be used as the unique $B g l \mathrm{II}$ site into which nucleotides are inserted to adjust spacing between the regulatory sequence and the promoter. Thus, insertion of synthetic BamHI-regulator- $B g l$ II cassettes provides a simple, single-step method to introduce any DNA sequence of choice into pGL3/TK in the desired orientation. Furthermore, the MCS can be used for direct insertion of restriction enzyme fragments excised from native DNA, and also for insertion of more than one regulatory sequence. There are cases where the chemicals to be tested directly affect the activity of reporter gene products, and in such cases the reporter gene may need to be substituted. Accordingly, the luciferase reporter gene in pGL3/TK can be excised and replaced with another reporter gene.

Specific Reporter Plasmids Based on pGL3/TK To evaluate the performance of the pGL3/TK plasmid, we constructed three specific reporter plasmids based on this plasmid. Three types of nuclear receptor target sites (Fig. 1), which have been reported in previous studies, were chosen as the regulatory DNA sequences to be inserted into pGL3/TK:

(a) ERE, the target sequence of the estrogen receptor, contains a palindrome of inverted AGGTCA repeats with a 3-base gap. We used the ERE of the Xenopus laevis vitellogenin A2 gene that completely matches the consensus sequence mentioned above, and has previously been used in transfection assays. $^{7,8)}$ It has been reported that a single copy of ERE is enough to induce an estrogen response, ${ }^{3)}$ and the ERE is also active in human cells. ${ }^{9)}$

(b) GRE, the target site of the glucocorticoid receptor, shares the same nucleotide sequence with the targets of the progesterone, androgen, and mineralcorticoid receptors (PRE, ARE, and MRE, respectively), and the consensus sequence includes a nearly perfect palindrome separated by a 3-bp gap. PRE copies located in the upstream region of the rat tyrosine aminotransferase gene ${ }^{10,11)}$ were used in the present study. It was reported that at least two copies of PRE are required to induce a Prog response. ${ }^{12)}$

(c) XRE, which is the target site of aryl hydrocarbon receptor, has a consensus core sequence of TNGCGTG. We used a region upstream of the mouse CYP1A1 gene $(-1282$ to -1212$)$ that contains two copies of XRE. ${ }^{4)}$

Performance of the pGL3/TK-Based Reporter Plasmids The reporter plasmids pGL3/ERE-TK, pGL3/(GRE) $)_{2}$ TK and pGL3/(XRE) $)_{2}$ TK, carrying ERE, GRE and XRE, respectively, were examined for their reporter expression in response to corresponding specific inducers. The cell line transfected with each plasmid is known to have a transcriptional regulation system via the corresponding DNA element, and has been frequently used for such investigations.

(a) Response of pGL3/ERE-TK to $\mathrm{E}_{2}$. MCF-7 cells that express estrogen receptor were transfected with pGL3/ERE-TK and treated with $\mathrm{E}_{2}$ (Fig. 2A). Reporter activity began to increase at $1 \mathrm{~nm} E_{2}$, and reached a maximum at $30 \mathrm{~nm}$, where reporter activity was elevated approximately 1.8 -fold. Changes in the ratio of FuGENE reagent: DNA did not improve the induction ratio. A synthetic hormone diethylstilbestrol showed a similar effect (data not shown).

(b) Response of pGL3/(GRE) $)_{2}$ TK to Dex. HeLa cells were transfected with pGL3/(GRE) $)_{2}-\mathrm{TK}$, and treated with a synthetic glucocorticoid, Dex (Fig. 2B). Reporter activity was markedly enhanced at $10 \mathrm{~nm}$ Dex, and reached a near
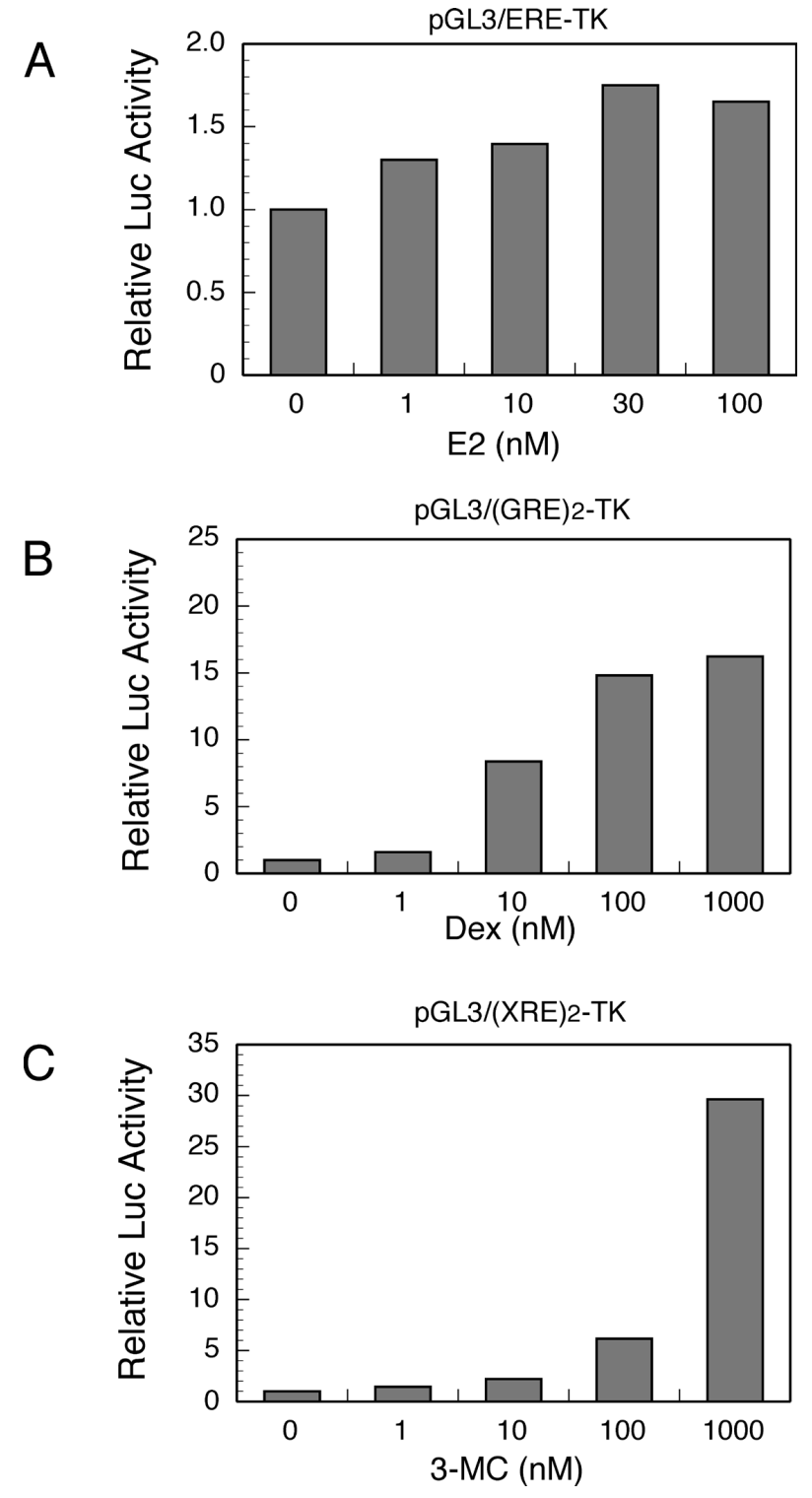

Fig. 2. Response of pGL3/TK-Based Reporter Plasmids to Corresponding Inducers

Relative luciferase (Luc) reporter activity was determined as described in Materials and Methods. Values are indicated as those relative to untreated controls (taken as 1). A, MCF-7 cells transfected with pGL3/ERE-TK were treated with the indicated concentrations of $E_{2}$. B, HeLa cells transfected with pGL3/(GRE) $)_{2}$-TK were treated with the indicated concentrations of Dex. C, HepG2 cells transfected with $\mathrm{pGL} 3 /(\mathrm{XRE})_{2}$-TK were treated with the indicated concentrations of 3-MC.

maximum at $100 \mathrm{~nm}$.

(c) Response of pGL3/(XRE) $)_{2}$ TK to 3-MC. HepG2 cells were transfected with $\mathrm{pGL} 3 /(\mathrm{XRE})_{2}-\mathrm{TK}$, and treated with 3-MC, an inducer of the CYP1A1 gene that encodes an enzyme involved in xenobiotic and drug metabolism. Dose-dependent induction of reporter activity was observed; induction was detectable at $1 \mathrm{~nm} 3-\mathrm{MC}$, and reporter activity continued to increase up to $1 \mu \mathrm{M}$ (Fig. 2C).

Reproducibility was verified by three independent experiments. These data confirmed that all the reporter plasmids tested are able to respond to cognate inducers in a concentration-dependent manner.

Response of pGL3/(GRE) $)_{2}$-TK to Different Inducers As mentioned above, we expected that pGL3/(GRE) $)_{2}$-TK would 

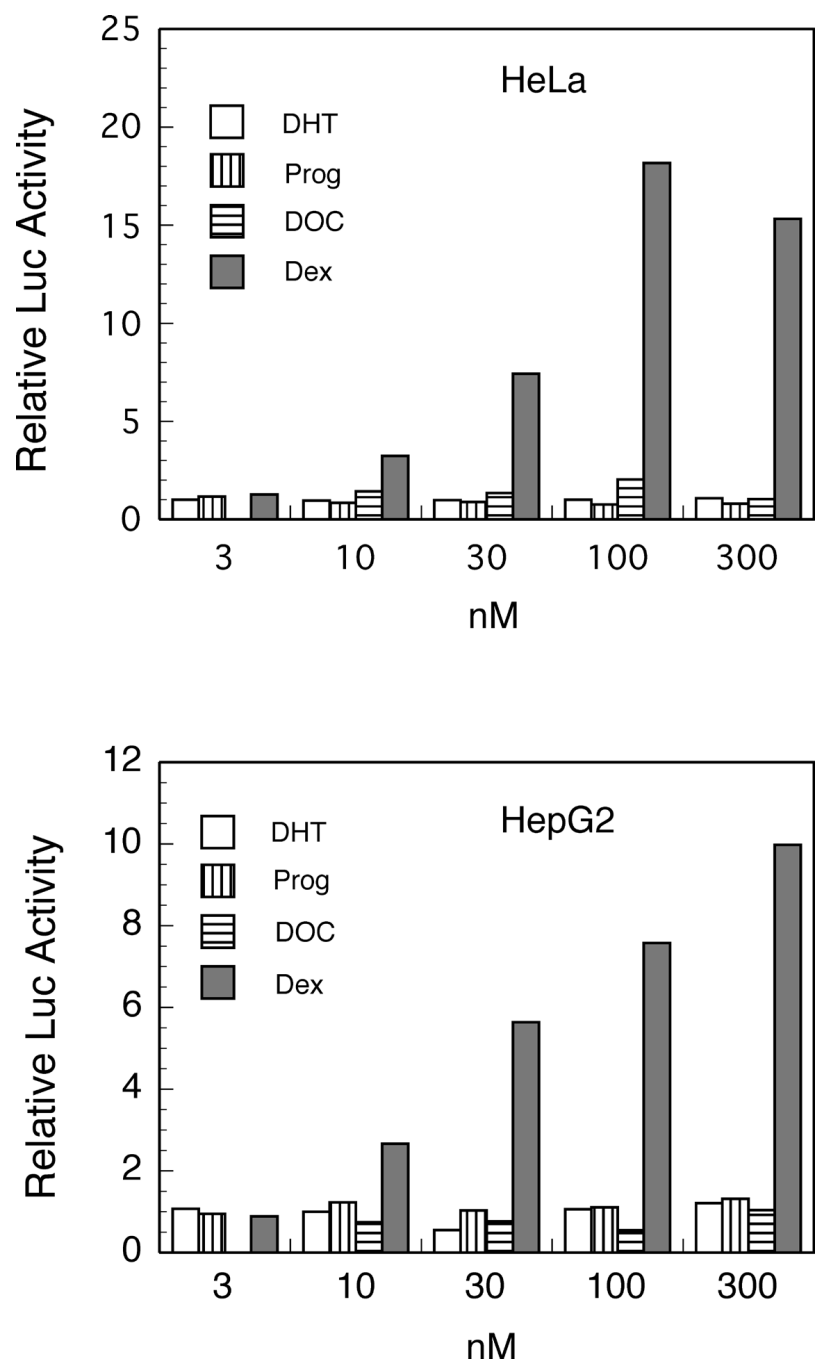

Fig. 3. Ligand-Specific Response of pGL3/(GRE) $)_{2}$ TK Reporter Plasmid

HeLa cells (upper panel) and HepG2 cells (lower panel) were transfected with pGL3/(GRE) $)_{2}$ TK, and the effects of the indicated concentrations of DHT, Prog, DOC and Dex were determined as described in Materials and Methods. The values indicated are relative to untreated controls (taken as 1).

respond to steroid hormones that regulate target genes via GRE, PRE, ARE and MRE. Thus, responses of this reporter plasmid to different hormones, i.e. Dex, Prog, DHT and DOC were examined. In this experiment two cell lines HeLa and HepG2, which could exhibit unique responses based on their own patterns of hormone receptor expression, were used (Fig. 3, upper and lower panels, respectively). In both cell types, reporter expression was activated only by Dex, and no response to Prog, DHT or DOC was observed. These results are likely to reflect the fact that while both cell types express the glucocorticoid receptor, they do not express receptors for other steroids. Thus, this system is able to detect a specific response to glucocorticoid in these cell types. We observed that the responses to Dex differed between the two cell lines. In HeLa cells, reporter activity began to increase at $10 \mathrm{~nm}$, and then sharply increased to reach a maximum at $100 \mathrm{~nm}$ (Fig. 3, upper panel). On the other hand, in HepG2 cells, reporter activity gently increased up to $300 \mathrm{~nm}$ (Fig. 3, lower panel). Reproducibility was confirmed by three independent experiments.

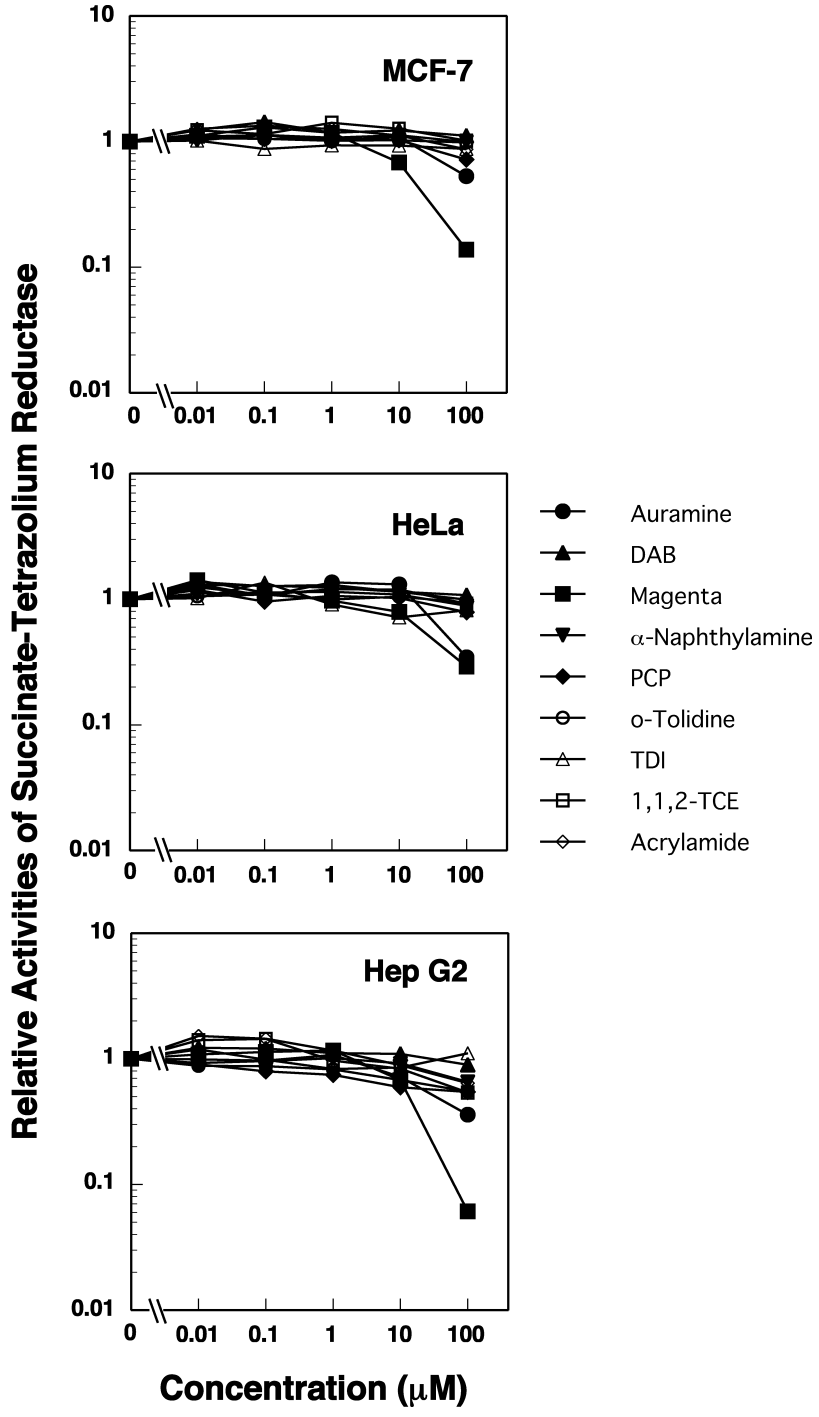

Fig. 4. Cytotoxicity of Industrial Chemicals

Indicated concentrations of chemicals were examined for cytotoxicity as described in Materials and Methods.

Evaluation of the Effects of Industrial Chemicals on Gene Regulation Mediated by Nuclear Receptor Target Sites We subsequently applied the transfection assay system to evaluate the effects of several industrial chemicals defined in "Ordinance on Prevention of Hazards Due to Specified Chemical Substances" in "Industrial Safety and Health Law" in Japan. These include auramine, DAB, magenta, $\alpha$-naphthylamine, PCP, $o$-tolidine, TDI, 1,1,2-TCE and acrylamide. Prior to the transfection assay, these chemicals were examined for cytotoxicity in MCF-7, HeLa and HepG2 cells by assessing a mitochondrial enzyme activity, which is an indicator of cell viability (Fig. 4). Dyes such as auramine and magenta showed moderate cytotoxicity at a concentration of $10 \mu \mathrm{M}$ in MCF-7 and HeLa cells, and obvious toxicity at $100 \mu \mathrm{M}$ in all cell lines. All chemicals, including the aforementioned dyes, had no effect at a concentration of $1 \mu \mathrm{M}$ or lower. Based on these results, transfection assays were performed within a concentration range up to $10 \mu \mathrm{M}$.

A preliminary screening assay was carried out using cells inoculated in 96-well microplates (1:5 scale on a cell number 
Table 1. Evaluation of the Effects of Industrial Chemicals on GRE- and XRE-Mediated Gene Regulation

\begin{tabular}{|c|c|c|c|c|c|}
\hline Assay $^{1)}$ & Chemical & $\mu \mathrm{M}$ & Relative activity ${ }^{2)}$ & $p$ Value $^{3)}$ & Induction \\
\hline \multirow[t]{4}{*}{ GRE } & Control $^{4)}$ & & $3.42 \pm 0.43$ & & 1.0 \\
\hline & $\mathrm{DAB}$ & 1 & $4.14 \pm 0.69$ & & 1.2 \\
\hline & & 10 & $5.14 \pm 0.37$ & $* * *$ & 1.5 \\
\hline & Dex & 0.1 & $38.90 \pm 9.08$ & $* * *$ & 11.4 \\
\hline \multirow[t]{8}{*}{$\mathrm{XRE}$} & Control $^{4)}$ & & $8.13 \pm 1.51$ & & 1.0 \\
\hline & DAB & 1 & $10.72 \pm 0.86$ & $*$ & 1.3 \\
\hline & & 10 & $17.59 \pm 2.49$ & $* * *$ & 2.2 \\
\hline & $\alpha$-Naphthylamine & 1 & $11.13 \pm 1.20$ & $*$ & 1.4 \\
\hline & & 10 & $14.93 \pm 2.76$ & $* *$ & 1.8 \\
\hline & $o$-Tolidine & 1 & $12.96 \pm 2.47$ & $* *$ & 1.6 \\
\hline & & 10 & $27.10 \pm 14.69$ & $*$ & 3.3 \\
\hline & $3-\mathrm{MC}$ & 1 & $131.13 \pm 1.01$ & $* * *$ & 16.1 \\
\hline
\end{tabular}

Effects of chemicals were determined in triplicates by the standard assay as described in Materials and Methods. ${ }^{1)}$ GRE, assay with the pGL3/(GRE) ${ }_{2}$-TK reporter; XRE, assay with the pGL3/(XRE) ${ }_{2}$-TK reporter. ${ }^{2)}$ (Firefly luciferase activity/Renilla luciferase activity) $\times 100$ with SE is indicated. ${ }^{3)}$ Two-tailed $p$-value against control: $* 0.05<p<0.1$; $* * 0.01<p<0.05 ; * * * p<0.01 .4) 0.05 \%$ DMSO was added to culture.

basis). From this assay, a weak but concentration-dependent increase in reporter activity was detected for DAB in the GRE assay and DAB, $\alpha$-naphthylamine and $o$-tolidine in the XRE assay (data not shown). The effects of these chemicals were further examined by the standard transfection assay using 24well plates, as shown in Table 1. As positive controls for GRE and XRE assays, $100 \mathrm{~nm}$ Dex and $1 \mu \mathrm{M} 3-\mathrm{MC}$ were included, respectively. Dimethylsulfoxide, which was used as solvent $(0.05 \%)$, was also included as a negative control, and assays were carried out in triplicate. In this experiment, all the chemicals tested resulted in statistically significant increases in reporter activity. DAB, at a concentration of $10 \mu \mathrm{M}$, increased GRE-mediated transcription by 1.5 -fold. Furthermore, $10 \mu \mathrm{M}$ of DAB, $\alpha$-naphthylamine and $o$-tolidine elevated XRE-mediated transcription by 2.2-, 1.8-, or 3.3-fold, respectively.

\section{DISCUSSION}

We constructed a versatile transfection assay system to permit evaluation of the effects of diverse industrial chemicals on gene regulation. A convenient, single-step insertion of a synthetic BamHI-regulator-BglII cassette into the basic pGL3/TK plasmid provides a simple and straightforward way to assay virtually any DNA sequence, and this can be used to evaluate the effects of a wide range of industrial chemicals. This method has the additional advantage that the insert orientation can be easily monitored, and also the distance from the TIS can be adjusted. Furthermore, the basic plasmid pGL3/TK was designed to have several useful properties: i) assays with a low background activity can be performed; ii) MCS serve as insertion sites for various restriction enzyme fragments; iii) the combined effects of more than one regulatory sequence can be evaluated by using multiple sites in MCS; and iv) the reporter gene can be exchanged if necessary. From the results of the present study, our transfection assay system is likely to work well; responses of three types of known regulatory sequences to corresponding inducers can successfully be detected, even without consideration of regulator/promoter spacing.

Nevertheless, some aspects could still be improved. The response of pGL3/ERE-TK to $E_{2}$ was low compared to that observed in previous reports; Vinggaard et al. reported between a 2- and 10-fold induction using the same cell line and transfection reagent. ${ }^{13)}$ It is known that the response of MCF-7 to estrogen differs between strains maintained in different laboratories, ${ }^{14)}$ and this may be affected by culture conditions as well as passage numbers. Use of alternative cell strains may be required in order to obtain better results. Although induction of GRE and XRE reporters by cognate inducers was consistently observed, induction ratios at the same inducer concentration varied between experiments. This was probably due to fluctuation of the low basal level values that were used to calculate induction ratios. Preliminary experiments showed that normalization of values relative to an appropriate internal positive control yielded less variable results, suggesting a solution for this problem.

In order to further improve the application of the transfection assay, establishment of a high-throughput procedure is likely to be important. For this purpose, a scale-downed version of the assay and simplification of the procedure may be necessary. We performed a small-scale assay using a 96-well microplate as preliminary screening, and the results obtained were roughly consistent with those of a standard assay with 24-well plates (data not shown). However, the 96-well assay still has problems in accuracy and reproducibility. Solutions for these points, for example, improving the accuracy of liquid handling, would make this assay more practical.

By testing the effects of industrial chemicals in this transfection assay system, DAB was observed to have a weak but significant effect on both GRE- and XRE-mediated transcription. It has been reported that DAB activates transcription via $\mathrm{XRE}$ in yeast with effective concentrations similar to those reported here. ${ }^{15}$ ) The present study confirmed such an effect of DAB directly in mammalian cells. The XRE assay also detected the positive effects of $\alpha$-naphthylamine and $o$-tolidine. Thus, these chemicals could disturb normal gene regulation, although their activity was much weaker than cognate inducers. We anticipate that the versatile transfection assay system established here will serve as a powerful tool to evaluate the effects of diverse industrial chemicals on gene regulation in future studies.

\section{REFERENCES}

1) Otsuka F. Gene expression assay for hazard assessment of 
chemicals. Ind. Health, 40, 113-120 (2002).

2) Koizumi S, Suzuki K, Ogra Y, Yamada H, Otsuka F. Transcriptional activity and regulatory protein binding of metal-responsive elements of the human metallothionein-IIA gene. Eur. J. Biochem., 259, 635-642 (1999).

3) Martinez E, Givel F, Wahli W. The estrogen-responsive element as an inducible enhancer: DNA sequence requirements and conversion to a glucocorticoid-responsive element. EMBO J., 6, 3719-3727 (1987).

4) Lusska A, Shen E, Whitlock JP Jr. Protein-DNA interactions at a dioxin-responsive enhancer. Analysis of six bona fide DNA-binding sites for the liganded Ah receptor. J. Biol. Chem., 268, 6575-6580 (1993).

5) Boonyaratanakornkit V, Melvin V, Prendergast P, Altmann M, Ronfani L, Bianchi ME, Taraseviciene L, Nordeen SK, Allegretto EA, Edwards DP. High-mobility group chromatin proteins 1 and 2 functionally interact with steroid hormone receptors to enhance their DNA binding in vitro and transcriptional activity in mammalian cells. Mol. Cell. Biol., 18, 4471-4487 (1998).

6) Kumar NS, Richer J, Owen G, Litman E, Horwitz KB, Leslie KK. Selective down-regulation of progesterone receptor isoform $\mathrm{B}$ in poorly differentiated human endometrial cancer cells: implications for unopposed estrogen action. Cancer Res., 58, 1860-1865 (1998).

7) Metzger D, White JH, Chambon P. The human oestrogen receptor functions in yeast. Nature, 334, 31-36 (1988).

8) Peale FV Jr, Ludwig LB, Zain S, Hilf R, Bambara RA. Properties of a high-affinity DNA binding site for estrogen receptor. Proc.
Natl. Acad. Sci. U.S.A., 85, 1038-1042 (1988).

9) Klein-Hitpass L, Schorpp M, Wagner U, Ryffel GU. An estrogen-responsive element derived from the $5^{\prime}$ flanking region of the Xenopus vitellogenin A2 gene functions in transfected human cells. Cell, 46, 1053-1061 (1986).

10) Tsai SY, Carlstedt-Duke J, Weigel NL, Dahlman K, Gustafsson JA, Tsai MJ, O'Malley BW. Molecular interactions of steroid hormone receptor with its enhancer element: evidence for receptor dimer formation. Cell, 55, 361-369 (1988).

11) Jantzen HM, Strähle U, Gloss B, Stewart F, Schmid W, Boshart M, Miksicek R, Schütz G. Cooperativity of glucocorticoid response elements located far upstream of the tyrosine aminotransferase gene. Cell, 49, 29-38 (1987).

12) Tsai SY, Tsai MJ, O'Malley BW. Cooperative binding of steroid hormone receptors contributes to transcriptional synergism at target enhancer elements. Cell, 57, 443-448 (1989).

13) Vinggaard AM, Joergensen EC, Larsen JC. Rapid and sensitive reporter gene assays for detection of antiandrogenic and estrogenic effects of environmental chemicals. Toxicol. Appl. Pharmacol., 155, 150-160 (1999).

14) Zacharewski T. In vitro bioassays for assessing estrogenic substances. Environ. Sci. Technol., 31, 613-623 (1997).

15) Kato TA, Matsuda T, Matsui S, Mizutani T, Saeki K. Activation of the aryl hydrocarbon receptor by methyl yellow and related congeners: structure-activity relationships in halogenated derivatives. Biol. Pharm. Bull., 25, 466-471 (2002). 